\title{
Open Water Test Series with Modified AU-Type Four-Bladed Propeller Models
}

\author{
By Atsuo Yazaki, Member* Einosuke Kuramochi, Member* \\ Tetsuaki Kumasaki, Member** \\ Summary
}

The paper reoords the result of experiments with a systematic series of modified AU-type four:bladed propeller models.

Charts of the series are shown by so called $\sqrt{B_{p}} \sim \delta$ design diagrams.

\section{Introduction}

Further to the work on AU-type five-bladed propellers, a paper read in the Society of Naval Architects of Japan (Vol.106), on the effect of propeller blades (Vol.103), and on AU and $A U_{w}$-type sixbladed propellers (Vol. 106), a systematic testing work with modified AU-type four-bladed propeller models for the solid-type propellers has been carried out in the Experiment Tank of Ship Propulsion Division.

In this report, authors write the result of this work, and give the design diagrams for the fourbladed propellers.

\section{Propeller Models}

List of the model propellers are given in Table 1.

Constructional diagrams and tables, stating all particulars of the blade sections of the modified AU-type series, are given in Fig. 1 and in the Tables 2 and 3 .

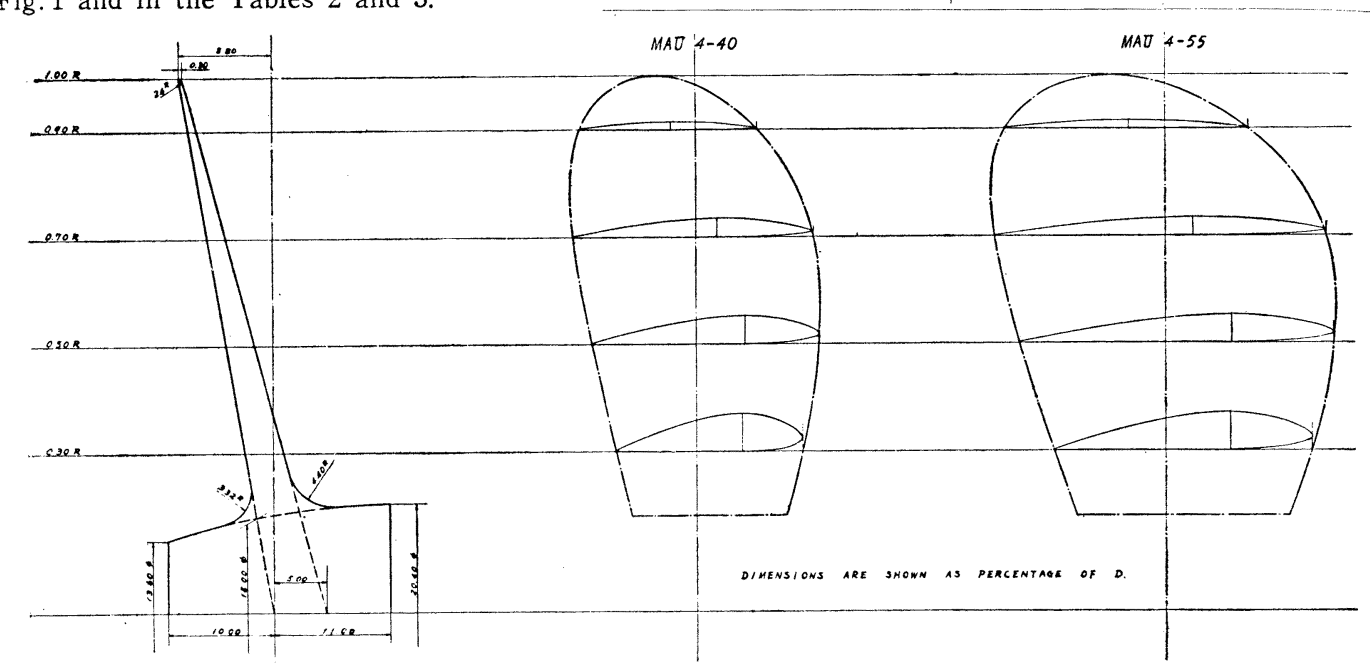

Fig. 1 General Plan of Modified AU 4 Series Propeller Models

原稿受付 昭和 35 年 6 月 20 日

* Transportation Technical Research Institute.

** Amagasaki Iron and Stee Mfg. Co. Ltd..

\begin{tabular}{l|c|c}
\hline & Modified AU4-40 & Modified AU4-55 \\
\hline M.P. NO. & $1305 \sim 1309$ & $1310 \sim 1314$ \\
Diameter (mm) & 250 & 250 \\
Boss Ratio & 0.180 & 0.180 \\
Exp. Area Ratio & 0.40 & 0.55 \\
Max. Blade Width Ratio & 0.226 & 0.311 \\
Mean Blade Width Ratio & 0.192 & 0.263 \\
Blade. Thickness Ratio & 0.050 & 0.050 \\
Angle of Rake & $10^{\circ}-0^{\prime}$ & $10^{\circ}-0^{\prime}$
\end{tabular}

Table 1 Particulars of Propeller Models 
Table 2 Dimensions of Modified AU-4 Series Prop.

\begin{tabular}{|c|c|c|c|c|c|c|c|c|c|c|c|c|c|}
\hline \multirow{4}{*}{ 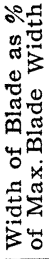 } & $r / R$ & 0.2 & 0.3 & 0.4 & 0.5 & 0.6 & 0.66 & 0.7 & 0.8 & 0.9 & 0.95 & 1.00 & \multirow{4}{*}{$\begin{array}{l}\text { Max. Blade } \\
\text { Width at } 0.66 \mathrm{R} \\
=0.226 \mathrm{D} \\
\text { for } F_{E} / F=0.40\end{array}$} \\
\hline & $\begin{array}{l}\text { From Gener- } \\
\text { ator Line to } \\
\text { TrailingEdge }\end{array}$ & 27.96 & 33.45 & 38.76 & 43.54 & 47.96 & 49.74 & 51.33 & 52.39 & 48.49 & 42.07 & 17.29 & \\
\hline & $\begin{array}{l}\text { From Gener- } \\
\text { ator Line to } \\
\text { Leading Edge }\end{array}$ & 38.58 & 44.25 & 48.32 & 50.80 & 51.15 & 50.26 & 48.31 & 40.53 & 25.13 & 13.55 & & \\
\hline & $\begin{array}{l}\text { Total Blade } \\
\text { Width }\end{array}$ & 66.54 & 77.70 & 87.08 & 94.34 & 99.11 & 100.00 & 99.64 & 92.92 & 73.62 & 55.62 & & \\
\hline \multicolumn{2}{|c|}{$\begin{array}{l}\text { Blade Thickness } \\
\text { as } \% \text { of } D\end{array}$} & 4.06 & 3.59 & 3.12 & 2.65 & 2.18 & 1.90 & 1.71 & 1.24 & 0.77 & 0.54 & 0.30 & $\begin{array}{l}\text { MaxBlade Thickn- } \\
\text { ess at Prop. Axis } \\
=0.050 \mathrm{D}\end{array}$ \\
\hline \multicolumn{2}{|c|}{$\begin{array}{l}\text { Distance of the Point } \\
\text { of Max.Thickness From } \\
\text { the Leading Edge as } \% \\
\text { of Blade Width }\end{array}$} & 32.0 & 32.0 & 32.0 & 32.5 & 34.9 & 37.9 & 40.2 & 45.4 & 48.9 & 50.0 & & \\
\hline
\end{tabular}

Table 3 Offsets of Modified AU Type Prop.

1) Ordinates of $X$ Value are Given as $\%$ of Blade Width.

2) Ordinates of $\boldsymbol{Y}$ Value are Given as $\%$ of $Y \max$.

\begin{tabular}{|c|c|c|c|c|c|c|c|c|c|c|c|c|c|c|c|c|c|}
\hline$r / R$ & $\begin{array}{l}X \\
Y_{0} \\
Y_{U}\end{array}$ & $\begin{array}{c}0 \\
35.00\end{array}$ & $\begin{array}{r}2.00 \\
51.85 \\
24.25\end{array}$ & $\begin{array}{r}4.00 \\
59.75 \\
19.05\end{array}$ & $\begin{array}{r}6.00 \\
66.15 \\
15.00 \\
\end{array}$ & $\begin{array}{l}10.00 \\
76.05 \\
10.00\end{array}$ & $\begin{array}{r}15.00 \\
85.25 \\
5.40\end{array}$ & $\begin{array}{r}20.00 \\
92.20 \\
2.35\end{array}$ & $\begin{array}{l}30.00 \\
99.80\end{array}$ & $\begin{array}{r}32.00 \\
100.00\end{array}$ & $\begin{array}{l}40.00 \\
97.75\end{array}$ & $\begin{array}{l}50.00 \\
89.95\end{array}$ & $\begin{array}{l}60.00 \\
78.15\end{array}$ & $\begin{array}{l}70.00 \\
63.15\end{array}$ & $\begin{array}{l}80.00 \\
45.25\end{array}$ & $\begin{array}{l}90.00 \\
25.30\end{array}$ & $\begin{array}{l}95.00100 .00 \\
15.00 \quad 4.50\end{array}$ \\
\hline 0.30 & $\begin{array}{l}X \\
Y_{0} \\
Y_{U}\end{array}$ & $\begin{array}{c}0 \\
35.00\end{array}$ & $\begin{array}{r}2.00 \\
51.85 \\
24.25\end{array}$ & $\begin{array}{r}4.00 \\
59.75 \\
19.05\end{array}$ & $\begin{array}{r}6.00 \\
66.15 \\
15.00\end{array}$ & $\begin{array}{l}10.00 \\
76.05 \\
10.00\end{array}$ & $\begin{array}{r}15.00 \\
85.25 \\
5.40 \\
\end{array}$ & $\begin{array}{r}20.00 \\
92.20 \\
2.35\end{array}$ & $\begin{array}{l}30.00 \\
99.80\end{array}$ & $\begin{array}{r}32.00 \\
100.00\end{array}$ & $\begin{array}{l}40.00 \\
97.75\end{array}$ & $\begin{array}{l}50.00 \\
89.95\end{array}$ & $\begin{array}{l}60.00 \\
78.15\end{array}$ & $\begin{array}{l}70.00 \\
63.15\end{array}$ & $\begin{array}{l}80.00 \\
45.25\end{array}$ & $\begin{array}{l}90.00 \\
25.30\end{array}$ & $\begin{array}{l}95.00100 .00 \\
15.00 \quad 4.50\end{array}$ \\
\hline 0.40 & $\begin{array}{l}X \\
Y_{0} \\
Y_{U}\end{array}$ & $\begin{array}{c}0 \\
35.00\end{array}$ & \begin{tabular}{|r|}
2.00 \\
51.85 \\
24.25 \\
\end{tabular} & $\begin{array}{r}4.00 \\
59.75 \\
19.05\end{array}$ & $\begin{array}{r}6.00 \\
66.15 \\
15.00\end{array}$ & $\begin{array}{l}10.00 \\
76.05 \\
10.00\end{array}$ & $\begin{array}{r}15.00 \\
85.25 \\
5.40 \\
\end{array}$ & $\begin{array}{r}20.00 \\
92.20 \\
2.35\end{array}$ & $\begin{array}{l}30.00 \\
99.80\end{array}$ & $\begin{array}{r}23.00 \\
100.00\end{array}$ & $\begin{array}{l}40.00 \\
97.75\end{array}$ & $\begin{array}{l}50.00 \\
89.95\end{array}$ & $\begin{array}{l}60.00 \\
78.15\end{array}$ & $\begin{array}{l}70.00 \\
63.15\end{array}$ & $\begin{array}{l}80.00 \\
45.25\end{array}$ & $\begin{array}{l}90.00 \\
25.30\end{array}$ & $\begin{array}{l}95.00100 .00 \\
15.00 \quad 4.50\end{array}$ \\
\hline 0.50 & $\begin{array}{l}X \\
Y_{0} \\
Y_{U}\end{array}$ & $\begin{array}{c}0 \\
35.00\end{array}$ & \begin{tabular}{|r|}
2.03 \\
51.85 \\
24.25 \\
\end{tabular} & $\begin{array}{r}4.06 \\
59.75 \\
19.05 \\
\end{array}$ & $\begin{array}{r}6.09 \\
66.15 \\
15.00 \\
\end{array}$ & $\begin{array}{l}10.16 \\
76.05 \\
10.00\end{array}$ & $\begin{array}{r}15.23 \\
85.25 \\
5.40\end{array}$ & $\begin{array}{r}20.31 \\
92.20 \\
23.5 \\
\end{array}$ & $\begin{array}{l}30.47 \\
99.80\end{array}$ & $\begin{array}{r}32.50 \\
100.00\end{array}$ & $\begin{array}{l}40.44 \\
97.75\end{array}$ & $\begin{array}{l}50.37 \\
89.95\end{array}$ & $\begin{array}{l}60.29 \\
78.15\end{array}$ & $\begin{array}{l}70.22 \\
63.15\end{array}$ & $\begin{array}{l}80.15 \\
45.25\end{array}$ & $\begin{array}{l}90.07 \\
25.30\end{array}$ & 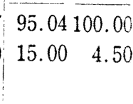 \\
\hline 0.60 & $\begin{array}{l}X \\
Y_{0} \\
Y_{U}\end{array}$ & $\begin{array}{c}0 \\
34.00\end{array}$ & $\begin{array}{r}2.18 \\
49.60 \\
23.60 \\
\end{array}$ & $\begin{array}{r}4.36 \\
58.00 \\
18.10\end{array}$ & $\begin{array}{r}6.54 \\
64.75 \\
14.25 \\
\end{array}$ & $\begin{array}{r}10.91 \\
75.20 \\
9.45\end{array}$ & $\begin{array}{r}16.36 \\
84.80 \\
5.00\end{array}$ & $\begin{array}{r}21.81 \\
91.80 \\
2.25 \\
\end{array}$ & $\begin{array}{l}32.72 \\
99.80\end{array}$ & $\begin{array}{r}34.90 \\
100.00\end{array}$ & $\begin{array}{l}42.56 \\
97.75\end{array}$ & $\begin{array}{l}52.13 \\
89.95\end{array}$ & $\begin{array}{l}61.70 \\
78.15\end{array}$ & $\begin{array}{l}71.28 \\
63.15\end{array}$ & $\begin{array}{l}80.85 \\
45.25\end{array}$ & $\begin{array}{l}90.43 \\
25.30\end{array}$ & $\begin{array}{l}95.21100 .00 \\
15.00 \quad 4.50\end{array}$ \\
\hline 0.70 & $\begin{array}{l}X \\
Y_{0} \\
Y_{U}\end{array}$ & $\begin{array}{c}0 \\
30.00\end{array}$ & $\begin{array}{r}2.51 \\
42.90 \\
20.50 \\
\end{array}$ & \begin{tabular}{r|}
5.03 \\
52.20 \\
15.45
\end{tabular} & $\begin{array}{r}7.54 \\
59.90 \\
11.95\end{array}$ & $\begin{array}{r}12.56 \\
71.65 \\
7.70\end{array}$ & $\begin{array}{r}18.84 \\
82.35 \\
4.10\end{array}$ & $\begin{array}{r}25.12 \\
90.60 \\
1.75\end{array}$ & $\begin{array}{l}37.69 \\
99.80\end{array}$ & $\begin{array}{r}40.20 \\
100.00\end{array}$ & $\begin{array}{l}47.23 \\
97.75\end{array}$ & $\begin{array}{l}56.03 \\
89.95\end{array}$ & $\begin{array}{l}64.82 \\
78.15\end{array}$ & $\begin{array}{l}73.62 \\
63.15\end{array}$ & $\begin{array}{l}82.41 \\
45.25\end{array}$ & $\begin{array}{l}91.21 \\
25.30\end{array}$ & $\begin{array}{l}95.60100 .00 \\
15.00 \quad 4.50\end{array}$ \\
\hline 0.80 & $\begin{array}{l}X \\
Y_{0} \\
Y_{U}\end{array}$ & $\begin{array}{c}0 \\
21.00\end{array}$ & \begin{tabular}{r|}
2.84 \\
32.45 \\
14.00 \\
\end{tabular} & \begin{tabular}{r|}
5.68 \\
41.70 \\
10.45
\end{tabular} & $\begin{array}{r}8.51 \\
50.10 \\
8.05 \\
\end{array}$ & $\begin{array}{r}14.19 \\
64.60 \\
5.05\end{array}$ & $\begin{array}{r}21.28 \\
78.45 \\
2.70\end{array}$ & $\begin{array}{r}28.38 \\
88.90 \\
1.15 \\
\end{array}$ & $\begin{array}{l}42.56 \\
99.80\end{array}$ & $\begin{array}{r}45.40 \\
100.00\end{array}$ & $\begin{array}{l}51.82 \\
97.75\end{array}$ & $\begin{array}{l}59.85 \\
89.95\end{array}$ & $\begin{array}{l}67.88 \\
78.15\end{array}$ & $\begin{array}{l}75.91 \\
63.15\end{array}$ & $\begin{array}{l}83.94 \\
45.25\end{array}$ & $\begin{array}{l}91.97 \\
25.30\end{array}$ & $\begin{array}{l}95.99100 .00 \\
15.00 \quad 4.50\end{array}$ \\
\hline 0.90 & $\begin{array}{l}X \\
Y_{0} \\
X_{U}\end{array}$ & $\begin{array}{l}0 \\
8.30\end{array}$ & $\begin{array}{r}3.06 \\
21.10 \\
4.00\end{array}$ & $\begin{array}{r}6.11 \\
31.50 \\
2.70\end{array}$ & $\begin{array}{r}9.17 \\
40.90 \\
2.05\end{array}$ & $\begin{array}{r}15.28 \\
57.45 \\
1.20\end{array}$ & $\begin{array}{r}22.92 \\
74.70 \\
0.70\end{array}$ & $\begin{array}{r}30.56 \\
87.45 \\
0.30 \\
\end{array}$ & $\begin{array}{l}45.85 \\
99.70\end{array}$ & $\begin{array}{r}48.90 \\
100.00\end{array}$ & $\begin{array}{l}54.91 \\
98.65\end{array}$ & $\begin{array}{l}62.42 \\
92.75\end{array}$ & $\begin{array}{l}69.94 \\
83.05\end{array}$ & $\begin{array}{l}77.46 \\
69.35\end{array}$ & $\begin{array}{l}84.97 \\
51.85\end{array}$ & $\begin{array}{l}92.49 \\
30.80\end{array}$ & 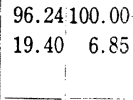 \\
\hline 0.95 & $\begin{array}{l}X \\
Y_{0} \\
Y_{U}\end{array}$ & $\begin{array}{l}0 \\
6.00\end{array}$ & $\begin{array}{r}3.13 \\
19.65\end{array}$ & $\begin{array}{r}6.25 \\
30.00\end{array}$ & $\begin{array}{r}9.38 \\
39.60\end{array}$ & $\begin{array}{l}15.63 \\
56.75\end{array}$ & $\begin{array}{l}23.44 \\
74.30\end{array}$ & $\begin{array}{l}31.25 \\
87.30\end{array}$ & $\begin{array}{l}46.87 \\
99.65\end{array}$ & $\begin{array}{r}50.00 \\
100.00\end{array}$ & $\begin{array}{l}55.88 \\
99.00\end{array}$ & $\begin{array}{l}63.23 \\
93.85\end{array}$ & $\begin{array}{l}70.59 \\
84.65\end{array}$ & $\begin{array}{l}77.94 \\
71.65\end{array}$ & $\begin{array}{l}85.30 \\
54.30\end{array}$ & $\begin{array}{l}92.65 \\
33.50\end{array}$ & $\begin{array}{l}96.32100 .00 \\
21.50 \quad 8.00\end{array}$ \\
\hline
\end{tabular}

The shape of profiles for modified AU-type series were improved from the AU-type series on. accounting of a prevention from cavitation of the back side and face side of the blade sections ${ }^{1)}$. Experiments have been done in two modified AU-type series of expanded-area ratio, namely 0.40 and 0.55 , in order to provide means of interpolation and extrapolation. As to pitch ratio, 0.5, 0.6, 0.8, 1.0 and 1.2 are tested. Pitch distribution is constant from the hub to the tip. All ten propeller models have a diameter of $250 \mathrm{~mm}$, and are made of alminium alloy.

\section{Open Water Tests}

The tests were carried out by means of a propeller open water test dynamometer (Mitsubishi-type), according to the scheme of the Experiment Tank of Ship Propulsion Division. The arrangement of the test instruments is shown in Fig. 2.

1) H. Takahashi ; A. Prevention from Face Cavitation by varying the Form of Blade Sections of a. Screw Propeller, Report of T. T. R. I. No. 38, 1959. 
All propeller models have been tested over a 100\% slip range at an immersion to the centre of propeller shaft equal to the screw diameter, and the number of revolutions was kept constant, namely 11.5 revolutions per second, and the speed of advance varied from zero to above the speed for zero thrust.

For fixing the net thrust, the correction for the resistance of the screw hub at various speed of advance was applied.

Mean temperature of water among these tests in $8.0^{\circ} \mathrm{C}$, and the Reynolds number according to the following formulas are as in Table 4 . So, it is considered scale effect is negligible.

$$
\begin{aligned}
& R_{n 1}=n D^{2} / v \\
& R_{n 2}=n D^{2} / v \cdot a_{E} / \boldsymbol{z} \\
& R_{n 3}=b_{m n} \cdot n D^{2} / v
\end{aligned}
$$

$n=$ Revolutions per second, $D=$ Diameter of screw, $a_{E}=$ Expanded-area ratio, $\quad z=$ Number of blades, $b_{m n}=$ Mean blade width ratio,

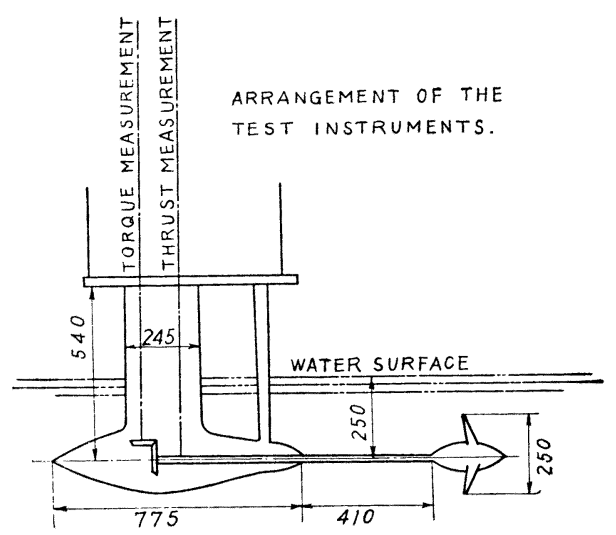

Fig. 2 Arrangement of the Test Instruments

$\nu=$ Kinematic viscosity coefficient, $R_{n}=$ Reynolds number.

Table 4 Reynolds number

\begin{tabular}{l|c|c|c}
\hline & Formula (1) & Formula (2) & Formula (3) \\
\hline Modified AU 4-40 & $5.21 \times 10^{5}$ & $5.21 \times 10^{4}$ & $1.00 \times 10^{5}$ \\
Modified AU 4-55 & $5.21 \times 10^{5}$ & $7.16 \times 10^{4}$ & $1.37 \times 10^{5}$ \\
\hline
\end{tabular}

\section{Diagrams}

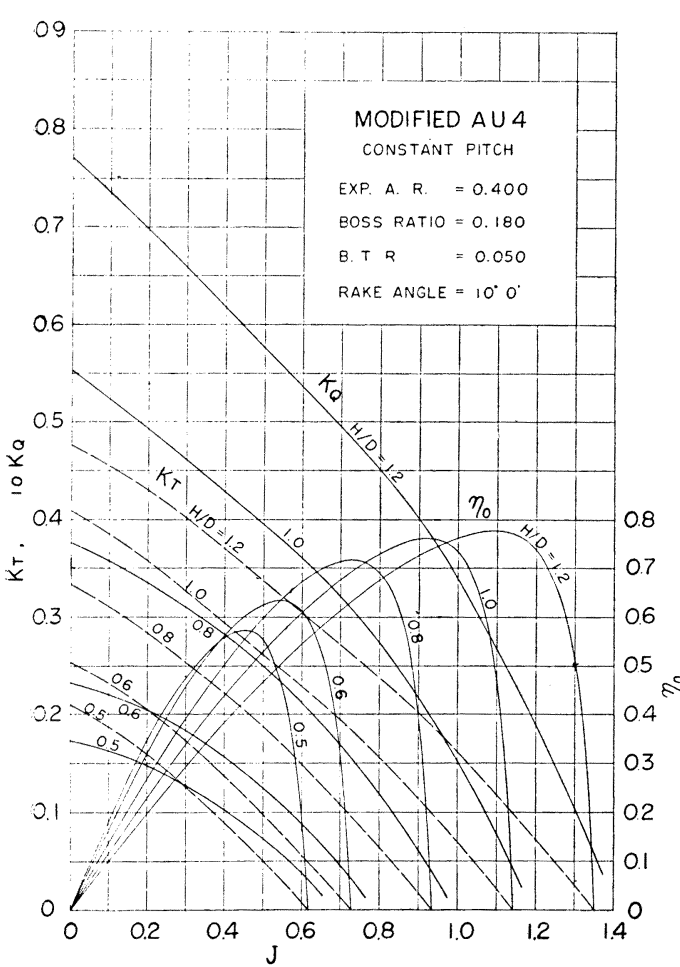

Fig. $3 J-K_{T}-K_{Q}$ Diagram (Modified AU 4-40)

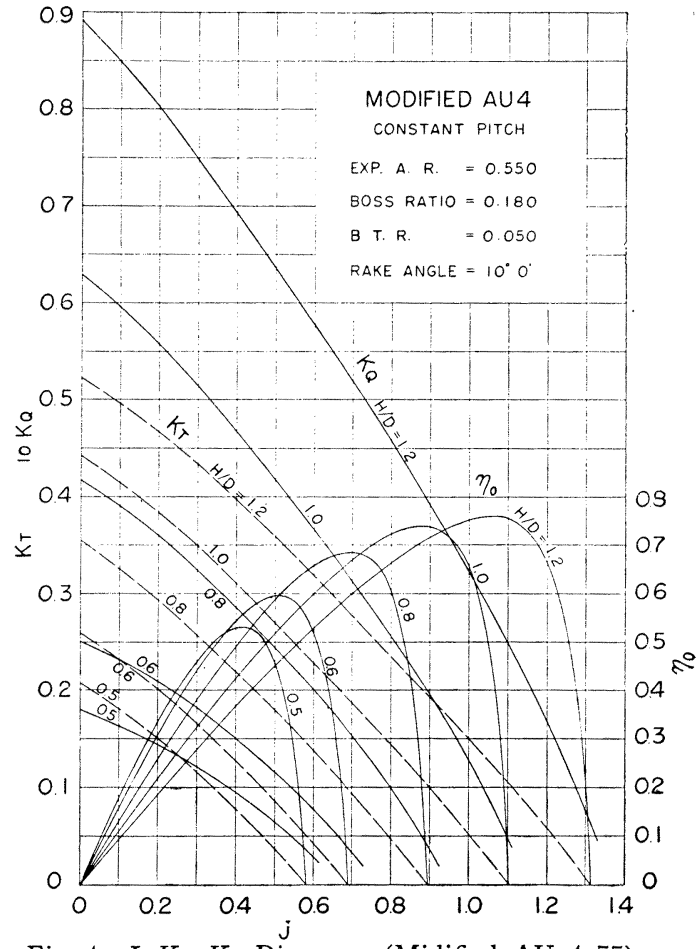

Fig. $4 J-K_{T}-K_{Q}$ Diagram (Midified AU 4-55) 


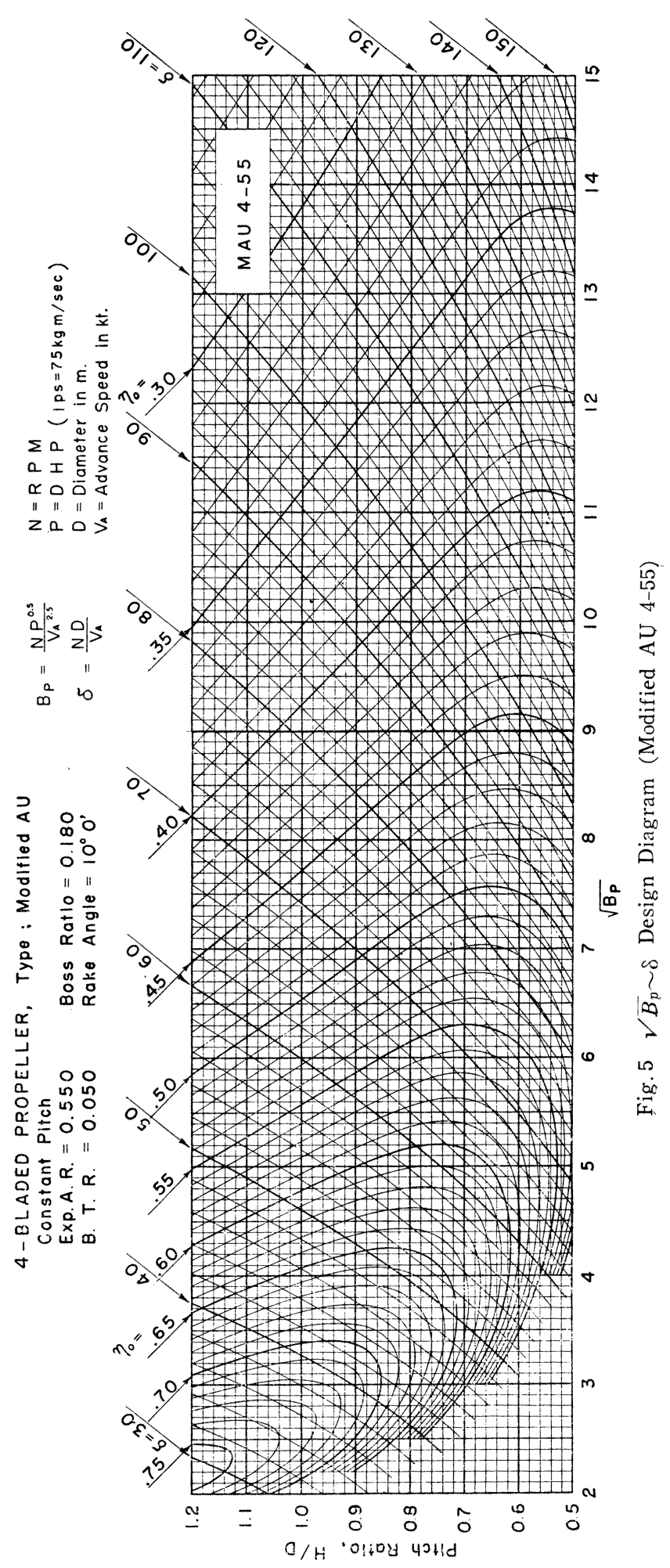




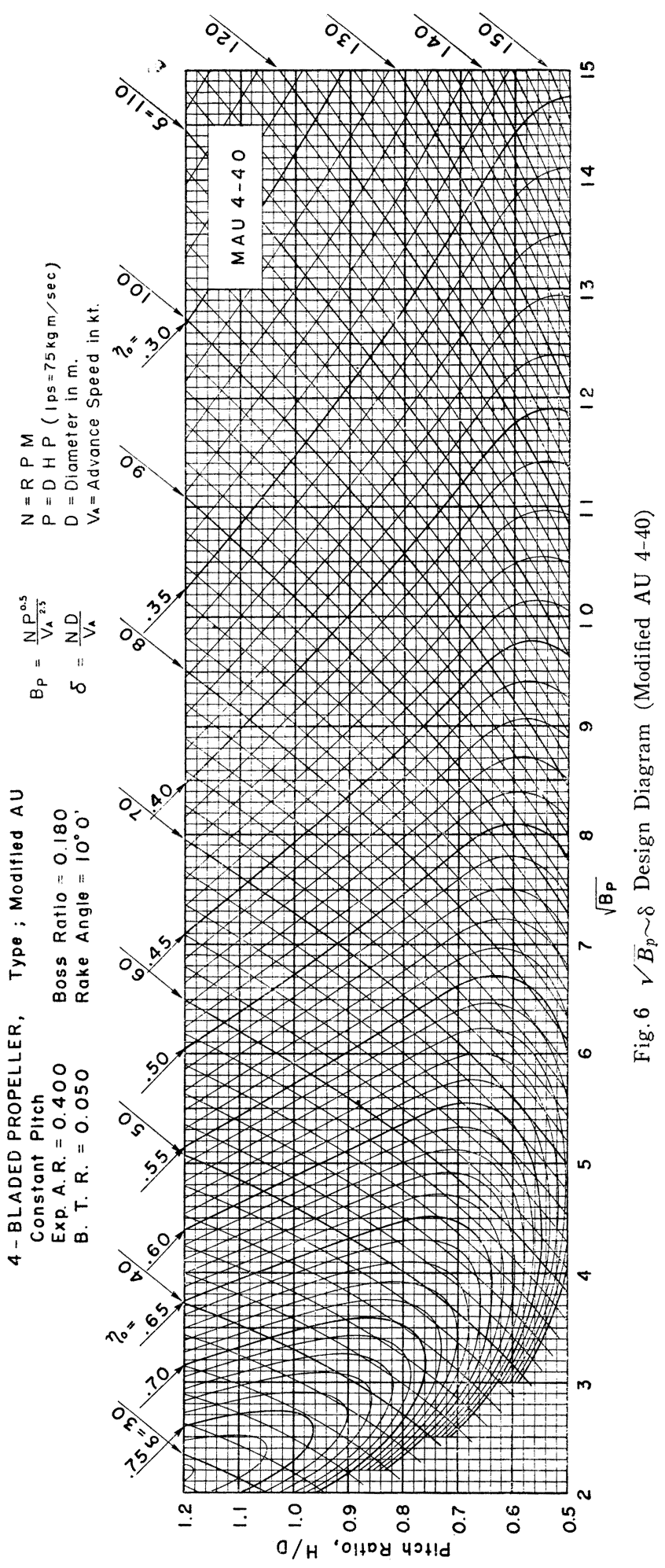


(a) Curves of $K_{T}, K_{Q}$ and $\eta_{0}$ on a base of $J$;

These curves are given in Figs. 3 and 4.

The meaning of symbols is as follows

$K_{T}=$ Thrust coefficient $=T / \rho n^{2} D^{4}$

$K_{Q}=$ Torque coefficient $=Q / \rho n^{2} D^{5}$

$\eta_{0}=$ Propeller efficiency in open water $=J \cdot K_{T} / 2 \pi K_{Q}$

$J=$ Advance coefficient $=v_{A} / n D$

$T=$ Measured thrust, $Q=$ Measured torque

$v_{A}=$ Speed of advance, $\rho=$ Density of tank water

(b) $\sqrt{B_{p}} \sim \delta$ design diagrams ;

In these diagrams, we used metric units, and density of sea water is assumed as $101 ., 51 \mathrm{~kg} \cdot \mathrm{m}^{-4} \cdot \mathrm{sec}^{-2}$. $\sqrt{B_{p}} \sim \delta$ design diagrams are given in Fig. 5 and 6.

The meaning of symbols is as follows :

$B_{p}=$ Power coefficient $=N P^{0.5} / V_{A}^{2.5}$

$\delta=$ Diameter constant $=N D / V_{A}$

$H / D=$ Pitch ratio, $N=$ Revolutions per minute for ship,

$P=$ Delivered horse power in sea water,

$V_{A}=$ Speed of advance through the wake water in knots, $D=$ Screw diameter in meter.

$1 P S=75 \mathrm{~kg} \cdot \mathrm{m} \cdot \mathrm{sec}^{-1}$

\section{Acknowledgement}

Authors wish to thank Mr. K. Tsuchida (chief of Ship Propulsion Division), and the staff of the Experiment Tank and the Amagasaki Iron and Steel Mfg. Co. Ltd., especially Mrs. Yoshiro Kawakami and Michio Takahashi, for their valuable advices in the evaluation of the experiments and the preparation of the propeller charts. 